\title{
SYMMETRIC AND UNSYMMETRIC BUCKLING OF CIRCULAR ARCHES
}

\author{
BY \\ R. W. DICKEY (Department of Mathematics, University of Wisconsin, Madison, WI) \\ AND \\ JOSEPH J. ROSEMAN (School of Mathematical Sciences, Tel Aviv University, Israel)
}

\begin{abstract}
A nonlinear geometrically exact inextensible elastica theory is used to derive a mathematical system which models a clamped circular arch of central angle $2 \alpha$ under the action of a vertical force field of amplitude $P$ (e.g., gravity). The equilibria of the arch are studied for various values of $\alpha, 0<\alpha<\pi$. The existence of a solution of symmetric form for all fixed values of $P$ and $\alpha$ is proved analytically by arguments based on variational principles. Numerical solutions are calculated for a variety of choices of $\alpha$, and in each case buckling (nonuniqueness) is shown to occur when $P$ is sufficiently large. In some cases, both symmetric and unsymmetric configurations are found, but each unsymmetric configuration obtained is found to be an unstable equilibrium, having energy greater than that of the symmetric configuration. Implications concerning the relative strengths and weaknesses of the various arches are discussed.
\end{abstract}

1. Introduction. The purpose of this paper is to discuss the equilibria of an arch under the action of a vertical force (e.g., gravity). It is assumed that the undeformed arch is some portion of a circular annulus and that the center-line of this annulus is inextensible. The theory that is derived under these assumptions (cf. Sec. 4) is a geometrically exact nonlinear theory which leads to the differential equation

$$
\frac{d^{2} \psi}{d \theta^{2}}+\lambda \theta \cos (\psi+\theta)+\mu \sin (\psi+\theta)+\omega \cos (\psi+\theta)=0
$$

with boundary condition

$$
\psi(-\alpha)=\psi(\alpha)=0
$$

(where $\alpha$ is some angle, $0<\alpha<\pi$ ) and constraints

$$
\begin{gathered}
\int_{-\alpha}^{\alpha} \cos (\psi+\theta) d \theta=2 \sin \alpha \\
\int_{-\alpha}^{\alpha} \sin (\psi+\theta) d \theta=0
\end{gathered}
$$

Received September 3, 1994.

1991 Mathematics Subject Classification. Primary 73K05. 
The dependent variable $\psi$ is the change in angle that the normal to the center-line makes before and after deformation. The parameters $\mu$ and $\omega$ are Lagrange multipliers to be used to satisfy the constraints (1.3) and

$$
\lambda=\frac{P a}{E I}
$$

where $P$ is the applied loading, $a$ is the radius of the center-line, $E$ is Young's modulus, and $I$ is a geometry parameter

$$
I=\iint_{A}\left(\frac{r}{a-r}\right)^{2} d A
$$

$A$ being the cross section of the arch.

The theory of arches, which is described by Eqs. (1.1), (1.2), and (1.3), is related to the various theories of rings, which are studied in [1], [2], and [3]. The behavior of the arch itself has been studied by Stoker [4, pp. 82-91]. The analysis of the arch presents difficulties, which do not occur for the ring. In the case of the ring the trivial solution exists for every value of $\lambda$. This is not true for the arch. Indeed, Eq. (1.1) has the trivial solution only in the case $\lambda=0$, i.e., in the case of zero load. Numerical evidence (cf. Sec. 3 ) indicates that, like the ring, for all values of $\alpha(0<\alpha<\pi)$ there is a critical load at which the arch buckles. However, unlike the ring, the arch undergoes some deformation before the critical load is reached. For some values of $\alpha$ this pre-buckling deformation may be quite large. In other cases it is extremely small.

A second feature that distinguishes the arch from the ring is the existence of unsymmetric solutions. The arch has unsymmetric solutions, while no unsymmetric solutions have been found for the ring. These results are discussed in Sec. 3. In Sec. 2 it is shown that (1.1), (1.2), and (1.3) have a solution for all values of $\lambda$. These equations are derived in the appendix (Sec. 4).

2. Existence theory. The mathematical problem considered in this section, which arises from our physical model, consists of showing the existence of a function $\psi(\theta) \in$ $C^{2}(-\alpha, \alpha) \cap C[-\alpha, \alpha]$, and two parameters $\mu$ and $\omega$ which together satisfy the system (1.1), (1.2), and (1.3) for every value of $\lambda$ on the interval $-\alpha<\theta<\alpha$. The proof of existence is accomplished by a variational approach. We associate with system (1.1), (1.2), and (1.3) the energy functional

$$
J[\psi]=\frac{1}{4} \int_{-\alpha}^{\alpha}\left[\psi^{\prime 2}-2 \lambda \theta \sin (\psi+\theta)\right] d \theta+\lambda(\sin \alpha-\alpha \cos \alpha)
$$

over the set of functions $Q$ that belong to $C^{1}(-\alpha, \alpha) \cap C[-\alpha, \alpha]$ and that also satisfy Eqs. (1.2) and (1.3). Equation (1.1) is then seen to be the Euler equation for $J[\psi]$ with Lagrange multipliers $\mu$ and $\omega$ arising from the integral constraints (1.3). We also see that $J=0$ when $\psi \equiv 0$ (which belongs to $Q$ ). The technique is to prove that a solution of the system (1.1), (1.2), and (1.3) exists by building a minimizing sequence of functions for $J[\psi]$, showing that the minimizing sequence converges to a limit function, and establishing that this limit function solves the problem.

In fact, this technique can be used to show the existence of an odd function solution; that is 
TheOREM 2.1. For every value of $\lambda$, there exist an odd function $\psi \in Q$ and a real number $\mu$ which solve system (1.1), (1.2), and (1.3) with $\omega=0$.

Proof. Every continuous odd function $\psi$ satisfies (1.3b) and if $\psi$ is also a solution of (1.1), then $\omega=0$, since the first three terms of (1.1) are odd functions while the fourth term, containing $\omega$, is an even function. It follows that for odd $\psi,(1.1),(1.2)$, and (1.3) reduce to the equivalent system below on the interval $0<\theta<\alpha$ :

$$
\begin{gathered}
\frac{d^{2} \psi}{d \theta^{2}}+\lambda \theta \cos (\psi+\theta)+\mu \sin (\psi+\theta)=0, \\
\psi(0)=\psi(\alpha)=0 \\
\int_{0}^{\alpha} \cos (\psi+\theta) d \theta=\sin \alpha
\end{gathered}
$$

and the energy functional may be expressed as

$$
J[\psi]=\int_{0}^{\alpha}\left[\frac{1}{2}{\psi^{\prime}}^{2}-\lambda \theta \sin (\psi+\theta)\right] d \theta+\lambda(\sin \alpha-\alpha \cos \alpha) .
$$

We shall work with (2.1) and (2.2). First, we choose $\left\{\sin \left(n \pi \frac{\theta}{\alpha}\right)\right\}_{n=1}^{\infty}$ as an orthogonal basis for $L_{2}(0, \alpha)$. For every integer $N \geq 2$, we consider the points $\mathbf{b}_{N}=\left(b_{1}, b_{2}, \ldots, b_{N}\right)$ in $N$-space and with each such point, we associate the function

$$
\psi_{N}(\theta)=\sum_{n=1}^{N} b_{n} \sin \left(n \pi \frac{\theta}{\alpha}\right), \quad 0<\theta<\alpha
$$

We next define

$$
C_{N}\left(\mathbf{b}_{N}\right)=\int_{0}^{\alpha} \cos \left(\psi_{N}+\theta\right) d \theta
$$

and

$$
H_{N}\left(\mathbf{b}_{N}\right)=\frac{\pi^{2}}{4 \alpha} \sum_{n=1}^{N} n^{2} b_{n}^{2}-\lambda \int_{0}^{\alpha} \theta \sin \left(\psi_{N}+\theta\right) d \theta+\lambda(\sin \alpha-\alpha \cos \alpha)
$$

We note that $H_{N}\left(\mathbf{b}_{N}\right)=J\left[\psi_{N}\right]$ and that $H_{N}(\mathbf{0})=0$. Now, we define $M=M(N)$ to be the set of points in $N$-space such that $C_{N}\left(\mathbf{b}_{N}\right)=\sin \alpha$ and $H_{N}\left(\mathbf{b}_{N}\right) \leq 1$. A point $\mathbf{b}_{N}$ in $M$ will be called a regular interior point if $H_{N}\left(\mathbf{b}_{N}\right)<1$ and $\nabla C_{N}\left(\mathbf{b}_{N}\right) \neq \mathbf{0}$.

We see that $C_{N}(\mathbf{0})=\sin \alpha, H_{N}(\mathbf{0})=0<1$, and that

$$
\begin{aligned}
\frac{\partial C}{\partial b_{j}}\left(\mathbf{b}_{N}\right) & =-\int_{0}^{\alpha} \sin \left(j \pi \frac{\theta}{\alpha}\right) \sin \left(\psi_{N}+\theta\right) d \theta, \quad j=1,2,3, \ldots, N \\
\frac{\partial C_{N}}{\partial b_{j}}(\mathbf{0}) & =-\int_{0}^{\alpha} \sin \left(j \pi \frac{\theta}{\alpha}\right) \sin \theta d \theta \\
& =\frac{\pi \alpha(-1)^{j} j \sin \alpha}{\pi^{2} j^{2}-\alpha^{2}} \neq 0, \quad j=1,2,3, \ldots, N
\end{aligned}
$$


for $0<\alpha<\pi$. Hence, $\mathbf{0}$ is a regular interior point of $M$ and so $M$, and indeed the set of regular interior points in $M$, is nonempty. From the definition, $M$ is seen to be closed and the condition that $H_{N}\left(\mathbf{b}_{N}\right) \leq 1$ implies that

$$
\sum_{n=1}^{N} b_{n}^{2} \leq \sum_{n=1}^{N} n^{2} b_{n}^{2} \leq \frac{2 \alpha^{3}|\lambda|+4 \alpha|\lambda|(1+\alpha)+4 \alpha}{\pi^{2}}=R(\alpha, \lambda)
$$

so that, for every fixed $\alpha$ and $\lambda$, the set $M$ is bounded. Thus, $M$ is a nonempty compact set. With $\mathbf{b}_{N}$ now being restricted to the set $M$, we have that $H_{N}\left(\mathbf{b}_{N}\right)$ is a continuous function on a nonempty compact set and therefore attains its minimum value in $M$, which value is nonpositive since $H_{N}(\mathbf{0})=0$. Let $\boldsymbol{\beta}_{N}=\left(\beta_{1}, \ldots, \beta_{N}\right)$ be a point at which $H_{N}$ attains its minimum and let

$$
\widetilde{\psi}_{N}(\theta)=\sum_{n=1}^{N} \beta_{n} \sin \left(n \pi \frac{\theta}{\alpha}\right) .
$$

From (2.4) and (2.5), it follows that if $\mathbf{b}_{N} \in \operatorname{Int}(M)$,

$$
d H_{N}\left(\mathbf{b}_{N}\right)=\sum_{n=1}^{N}\left[\frac{\pi^{2}}{2 \alpha} n^{2} b_{n}-\lambda \int_{0}^{\alpha} \theta \cos \left(\psi_{N}+\theta\right) \sin \left(n \pi \frac{\theta}{\alpha}\right) d \theta\right] d b_{n}
$$

and

$$
d C_{N}\left(\mathbf{b}_{N}\right)=-\sum_{n=1}^{N}\left[\int_{0}^{\alpha} \sin \left(\psi_{N}+\theta\right) \sin \left(n \pi \frac{\theta}{\alpha}\right) d \theta\right] d b_{n}=0 .
$$

Now, if it should be true that $\boldsymbol{\beta}_{N}$ is a regular interior point, then

$$
\begin{aligned}
& d H_{N}\left(\boldsymbol{\beta}_{n}\right)=\sum_{n=1}^{N}\left[\frac{\pi^{2}}{2 \alpha} n^{2} \beta_{n}-\lambda \int_{0}^{\alpha} \theta \cos \left(\widetilde{\psi}_{N}+\theta\right) \sin \left(n \pi \frac{\theta}{\alpha}\right) d \theta\right] d b_{n}=0, \\
& d C_{N}\left(\boldsymbol{\beta}_{n}\right)=-\sum_{n=1}^{N}\left[\int_{0}^{\alpha} \sin \left(\tilde{\psi}_{N}+\theta\right) \sin \left(n \pi \frac{\theta}{\alpha}\right) d \theta\right] d b_{n}=0
\end{aligned}
$$

and, therefore, for any value $\mu_{N}$, we have

$$
\begin{array}{r}
d H_{N}+\mu_{N} d C_{N}=\sum_{n=1}^{N}\left[\frac{\pi^{2}}{2 \alpha} n^{2} \beta_{n}-\lambda \int_{0}^{\alpha} \theta \cos \left(\widetilde{\psi}_{N}+\theta\right) \sin \left(n \pi \frac{\theta}{\alpha}\right) d \theta\right. \\
\left.\quad-\mu_{N} \int_{0}^{\alpha} \sin \left(\widetilde{\psi}_{N}+\theta\right) \sin \left(n \pi \frac{\theta}{\alpha}\right) d \theta\right] d b_{n}=0 .
\end{array}
$$

The assumption that $\boldsymbol{\beta}_{N}$ is a regular interior point also implies that there exists an integer $k, k=k(N), 1 \leq k \leq N$, such that $\int_{0}^{\alpha} \sin \left(\tilde{\psi}_{N}+\theta\right) \sin \left(k \pi \frac{\theta}{\alpha}\right) d \theta \neq 0$ (cf. (2.6)). We may therefore choose

$$
\mu_{N}=\frac{\frac{\pi^{2}}{2 \alpha} k^{2} \beta_{k}-\lambda \int_{0}^{\alpha} \theta \cos \left(\widetilde{\psi}_{N}+\theta\right) \sin \left(k \pi \frac{\theta}{\alpha}\right) d \theta}{\int_{0}^{\alpha} \sin \left(\widetilde{\psi}_{N}+\theta\right) \sin \left(k \pi \frac{\theta}{\alpha}\right) d \theta}
$$


and then, from the independence of the $N-1$ variables $d b_{n}, n \neq k$, it would follow that, for all $n=1,2,3, \ldots, N$,

$$
\frac{\pi^{2}}{2 \alpha} n^{2} \beta_{n}-\lambda \int_{0}^{\alpha} \theta \cos \left(\widetilde{\psi}_{N}+\theta\right) \sin \left(n \pi \frac{\theta}{\alpha}\right) d \theta-\mu_{N} \int_{0}^{\alpha} \sin \left(\widetilde{\psi}_{N}+\theta\right) \sin \left(n \pi \frac{\theta}{\alpha}\right) d \theta=0
$$

and also that

$$
C_{N}\left(\boldsymbol{\beta}_{N}\right)=\sin \alpha
$$

Equations $(2.16),(2.17)$ comprise a system of $N+1$ equations that $\beta_{1}, \beta_{2}, \ldots, \beta_{N}$ and $\mu_{N}$ must satisfy if the condition that $\nabla C_{N}\left(\boldsymbol{\beta}_{N}\right) \neq 0$ holds.

We shall now prove the following:

LEMmA 2.1. There exists a monotonic increasing subsequence $N_{j}$ and a fixed integer $k$, independent of $j$, such that if one denotes

$$
\boldsymbol{\beta}_{N_{j}}=\left(\beta_{1}\left(N_{j}\right), \beta_{2}\left(N_{j}\right), \ldots, \beta_{N_{j}}\left(N_{j}\right)\right)
$$

and

$$
\widetilde{\psi}_{N_{j}}(\theta)=\sum_{n=1}^{N_{j}} \beta_{n}\left(N_{j}\right) \sin \left(n \pi \frac{\theta}{\alpha}\right)
$$

then, for all $j$ sufficiently large,

$$
\int_{0}^{\alpha} \sin \left(\widetilde{\psi}_{N_{j}}+\theta\right) \sin \left(k \pi \frac{\theta}{\alpha}\right) d \theta \neq 0
$$

Proof. For convenience, we define $\beta_{n}(N)=0$ for $n>N$. Thus,

$$
\widetilde{\psi}_{N}(\theta)=\sum_{n=1}^{\infty} \beta_{n}(N) \sin \left(n \pi \frac{\theta}{\alpha}\right)
$$

From (2.8), we see that, for fixed $\alpha$ and $\lambda$,

$$
\sum_{n=1}^{\infty} \beta_{n}^{2}(N) \leq \sum_{n=1}^{\infty} n^{2} \beta_{n}^{2}(N) \leq R(\alpha, \lambda)
$$

Hence, $\left\{\beta_{1}(N)\right\}_{N=1}^{\infty}$ is a sequence that is bounded from above and below. Therefore, there exists a subsequence $N_{j}^{1}(j=1,2,3, \ldots)$ such that $\beta_{1}\left(N_{j}^{1}\right) \rightarrow \beta_{1}^{*}$ as $j \rightarrow \infty$. Now, $\beta_{2}\left(N_{j}^{1}\right)$ is also bounded from above and below and so there exists a subsequence $N_{j}^{2}$ of $N_{j}^{1}$ such that $\beta_{2}\left(N_{j}^{2}\right) \rightarrow \beta_{2}^{*}$ as $j \rightarrow \infty$ and it follows also that $\beta_{1}\left(N_{j}^{2}\right) \rightarrow \beta_{1}^{*}$ as $j \rightarrow \infty$. In a similar manner, we construct $N_{j}^{m}$ such that $\beta_{p}\left(N_{j}^{m}\right) \rightarrow \beta_{p}^{*}$ as $j \rightarrow \infty$ for $p \leq m$. We now consider the subsequence $N_{j}=N_{j}^{j}$ and we see that $\beta_{p}\left(N_{j}\right) \rightarrow \beta_{p}^{*}$ for all $p$ as $j \rightarrow \infty$. We next define

$$
\psi_{(\theta)}^{*}=\sum_{n=1}^{\infty} \beta_{n}^{*} \sin \left(n \pi \frac{\theta}{\alpha}\right)
$$


and we claim that $\widetilde{\psi}_{N_{j}}(\theta) \rightarrow \psi^{*}(\theta)$ uniformly on the interval $0<\theta<\alpha$ as $j \rightarrow \infty$. The proof of this claim is established as follows:

$$
\begin{aligned}
\left|\widetilde{\psi}_{N_{j}}-\psi^{*}\right| \leq & \sum_{n=1}^{L}\left|\beta_{n}\left(N_{j}\right)-\beta_{n}^{*}\right|+\sum_{n=L+1}^{\infty}\left(\left|\beta_{n}\left(N_{j}\right)\right|+\left|\beta_{n}^{*}\right|\right) \\
\leq & \sum_{n=1}^{L}\left|\beta_{n}\left(N_{j}\right)-\beta_{n}^{*}\right|+\left[\left(\sum_{n=L+1}^{\infty} n^{2}\left|\beta_{n}\left(N_{j}\right)\right|^{2}\right)^{1 / 2}+\left(\sum_{n=L+1}^{\infty} n^{2}\left|\beta_{n}^{*}\right|^{2}\right)^{1 / 2}\right] \\
& \cdot\left(\sum_{n=L+1}^{\infty} \frac{1}{n^{2}}\right)^{1 / 2} \\
\leq & \sum_{n=1}^{L}\left|\beta_{n}\left(N_{j}\right)-\beta_{n}^{*}\right|+2[R(\alpha, \lambda)]^{1 / 2}\left[\sum_{n=L+1}^{\infty} \frac{1}{n^{2}}\right]^{1 / 2}
\end{aligned}
$$

By first choosing $L$ sufficiently large and then choosing $j$ sufficiently large relative to $L$, we can make the right-hand side of $(2.24)$ as small as desired.

Since the convergence is uniform, the limit function $\psi^{*}(\theta)$ is continuous and

$$
\lim _{j \rightarrow \infty} C_{N_{j}}\left(\boldsymbol{\beta}_{N_{j}}\right)=\int_{0}^{\alpha} \cos \left(\psi^{*}+\theta\right) d \theta=\sin \alpha
$$

Equation (2.25) implies that $\psi^{*}+\theta \not \equiv m \pi\left(m\right.$ integer) and hence $\sin \left(\psi^{*}+\theta\right) \not \equiv 0$. Therefore, there exists an integer $k$ such that

$$
\int_{0}^{\alpha} \sin \left(k \pi \frac{\theta}{\alpha}\right) \sin \left(\psi^{*}+\theta\right) d \theta \neq 0
$$

and, accordingly, for all $j$ sufficiently large,

$$
\int_{0}^{\alpha} \sin \left(k \pi \frac{\theta}{\alpha}\right) \sin \left(\widetilde{\psi}_{N_{j}}+\theta\right) d \theta \neq 0 .
$$

We have established that (2.15) and (2.16) hold when $N=N_{j}$ and $j$ is sufficiently large. Letting $j \rightarrow \infty$ in (2.15) and (2.16) yields

$$
\mu^{*}=\frac{\frac{\pi^{2}}{2 \alpha} k^{2} \beta_{k}^{*}-\lambda \int_{0}^{\alpha} \theta \cos \left(\psi^{*}+\theta\right) \sin \left(k \pi \frac{\theta}{\alpha}\right) d \theta}{\int_{0}^{\alpha} \sin \left(\psi^{*}+\theta\right) \sin \left(k \pi \frac{\theta}{\alpha}\right) d \theta}
$$

and

$$
\begin{aligned}
\frac{\pi^{2}}{2 \alpha} n^{2} \beta_{n}^{*} & -\lambda \int_{0}^{\alpha} \theta \cos \left(\psi^{*}+\theta\right) \sin \left(n \pi \frac{\theta}{\alpha}\right) d \theta \\
& -\mu^{*} \int_{0}^{\alpha} \sin \left(\psi^{*}+\theta\right) \sin \left(n \pi \frac{\theta}{\alpha}\right) d \theta=0
\end{aligned}
$$


for every positive integer $n$. In addition, $\tilde{\psi}_{N_{j}}(\theta)$ is a minimizing function for the functional $J$ over $Q\left(N_{j}\right)$, where $Q\left(N_{j}\right)$ is the set of functions $\psi_{N_{j}}$ (cf. (2.3)) with a coefficient vector $\mathbf{b}_{N_{j}} \in M\left(N_{j}\right)$. Therefore, $\psi^{*}$ minimizes $J$ over $Q^{*}$ which is the subset of $L_{2}^{1}(-\alpha, \alpha) \cap$ $C[-\alpha, \alpha]$ containing odd functions that satisfy (2.1b) and (2.1c) or, equivalently, (1.2) and (1.3a).

There remains to prove that $\psi^{*}$ is twice differentiable on $(0, \alpha)$ and is a solution of (2.1a). This will now be done with the aid of a well-known Green's function:

$$
G(\theta, \phi)=\frac{1}{\alpha} \phi \theta-\min (\phi, \theta), \quad 0 \leq \phi, \theta \leq \alpha .
$$

(This Green's function vanishes at the endpoints and satisfies the equation

$$
\left.\frac{\partial^{2}}{\partial \theta^{2}} G(\theta, \phi)=\delta(\theta-\phi) .\right)
$$

We consider the integral operator

$$
I[\psi]=\int_{0}^{\alpha} G(\theta, \phi) F(\phi, \psi(\phi)) d \phi
$$

where

$$
F(\phi, \psi(\phi))=-\lambda \phi \cos (\psi(\phi)+\phi)-\mu^{*} \sin (\psi(\phi)+\phi)
$$

If we define

$$
h(\theta)=\psi^{*}(\theta)-I\left[\psi^{*}\right],
$$

then, for every $n=1,2,3, \ldots$, we discover that

$$
\begin{gathered}
\int_{0}^{\alpha} \sin \left(n \pi \frac{\theta}{\alpha}\right) h(\theta) d \theta=\frac{\alpha}{2} \beta_{n}^{*}-\frac{\lambda \alpha^{2}}{n^{2} \pi^{2}} \int_{0}^{\alpha} \theta \cos \left(\psi^{*}+\theta\right) \sin \left(n \pi \frac{\theta}{\alpha}\right) d \theta \\
-\frac{\mu^{*} \alpha^{2}}{n^{2} \pi^{2}} \int_{0}^{\alpha} \sin \left(\psi^{*}+\theta\right) \sin \left(n \pi \frac{\theta}{\alpha}\right) d \theta=0
\end{gathered}
$$

(cf. $(2.29))$ and thus $h(\theta) \equiv 0$ so that

$$
\psi^{*}(\theta)=I\left[\psi^{*}\right]
$$

Equation (2.36) implies that $\psi^{*}$ is twice differentiable and satisfies (2.1a) with $\mu=\mu^{*}$. This completes the proof of Theorem 2.1.

We note that for $\lambda \neq 0$, it is clear that $\psi^{*}(\theta) \not \equiv 0$ since $\psi(\theta) \equiv 0$ is not a solution of (2.1a) for any value of $\mu$.

We now redirect our attention to system (1.1), (1.2), and (1.3) and the functional $J$ as given in (2.0) over the interval $-\alpha<\theta<\alpha$. In a manner similar to the proof of Theorem 2.1, it is possible to prove the following: 
THEOREM 2.2. For every value of $\lambda$, there exist a function $\psi \in Q$ and a pair of real numbers $\mu, \omega$ that solve system (1.1), (1.2), and (1.3), and the function $\psi$ also minimizes $J[\psi]$ over $Q$.

The solution $\psi$ of (1.1), (1.2), and (1.3) that is obtained in the proof of Theorem (2.2) minimizes $J$ over all the functions in $Q$, as opposed to the solution $\psi$ of Theorem 2.1 that minimizes $J$ over the odd functions in $Q$. In every case treated numerically, the two solutions coincided; that is, the odd solution of Theorem 2.1 was also the solution of Theorem 2.2. In several cases, a non-odd solution of system (2.1) was found numerically, in addition to the odd solution. However, in each of these cases, the non-odd solution had a larger energy $J[\psi]$. The questions as to whether the odd solution of the system is always the minimum energy solution and as to which values of the parameters $\lambda$, $\mu$, and $\omega$ yield a unique solution are still under investigation.

3. Numerical solutions. Equation (1.1) with boundary conditions (1.2) was solved numerically for both the case of symmetric deformation $(\omega=0)$ and the case of unsymmetric deformation $(\omega \neq 0)$. In both cases the boundary value problem was solved by the "shooting method" (cf. [5]). The parameter $\mu$, and in the unsymmetric case $\omega$, was determined by Newton's Method to satisfy the constraints (1.3).

The problem was solved for a variety of choices of $\alpha$ with $0<\alpha \leq \pi$. Once $\psi$ is found the actual shape of the arch can be determined by quadrature (cf. Sec. 4). We will describe the results for three representative cases, in particular, $\alpha=\pi / 4, \alpha=\pi / 2$, and $\alpha=3 \pi / 4$. In Figures 3.1a, b, and c the solid curves are a plot of $\mu$ versus $\lambda$ for the case $\omega=0$, i.e., the symmetric case. The dashed curves in the figures are the projection of the $\lambda, \mu, \omega$ curve onto the $(\lambda, \mu)$-plane. Thus the dashed curve corresponds to unsymmetric solutions. The small circles on the diagrams indicate bifurcation points. Any point of intersection that is not enclosed in a circle is not a bifurcation point. The numbers on the diagrams in Figures 3.1a, b, and c refer to Figures 3.2, 3.3, and 3.4. In Figures 3.2, 3.3 , and 3.4 (see pp. 768-770) we have drawn equilibrium positions for the arch under various loads. In the figures the loads are acting in the direction of the negative $y$ axis. For example, (1) is the undeformed arch, i.e., it corresponds to $\lambda=0, \mu=0, \omega=0$. The values of the parameters for the states (1)-(9) are given in Table 3.1 (see p. 768).

Once problem (1.1)-(1.3) was solved numerically there was no difficulty in calculating the corresponding energy. In none of the cases that were studied was the unsymmetric solution the solution of minimum energy. However, if we assume the arch always takes up the position of minimum energy, buckling occurred in every case. The buckling was always from one symmetric state to another symmetric state. In particular, consider the case of decreasing $\lambda$ for the three different cases described in Figure 3.1. Assuming the arch takes up the position of minimum energy, the deformation is continuous from state (1) to state (2), the arch jumps from state (2) to state (3) (buckling) and for smaller values of $\lambda$ the deformation is again continuous, i.e., the arch deforms continuously from state (3) to state (4) and beyond. Thus in Figures 3.2 through 3.4, cases (1)-(4) are stable equilibria and (5)-(9) are unstable equilibria.

One feature that stands out in the behavior is the relative strength and weakness of the various arches. The buckling loads for the three different cases are $(\alpha=\pi / 4, \lambda \approx-18)$, 


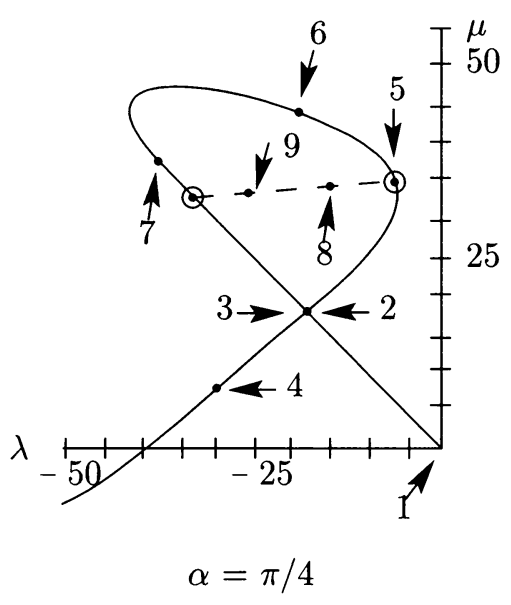

(a)

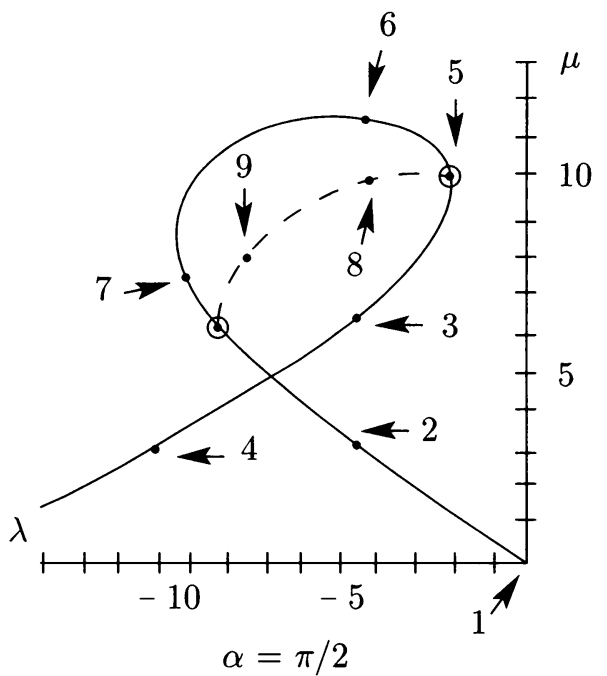

(b)

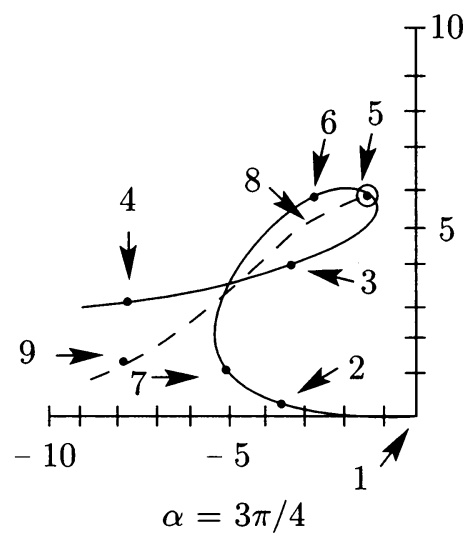

(c)

FIG. 3.1

$(\alpha=\pi / 2, \lambda \approx-5)$, and $(\alpha=3 \pi / 4, \lambda \approx-3.5)$. Thus the buckling load for an arch that is a quarter of a circle is much larger than either the half circle or the arch that is three-quarters of a circle. In addition, in the case $\alpha=3 \pi / 4$ the arch undergoes a large deformation even before the buckling load is reached. This arch is very flexible. It would be a poor choice for use in a structure since the relative flexibility would have to be compensated for by stiffer (and probably heavier) construction.

Because of the large deformation that was encountered in the case $\alpha=3 \pi / 4$ under relatively light loads, it was of interest to look at the extreme case $\alpha=\pi$. This would correspond to the case of a ring welded to the top of a column. In Figure 3.5 (see p. 771) a couple of symmetric equilibria and an unsymmetric equilibrium are drawn. As is to be expected, small values of $\lambda$ lead to large deformations. 


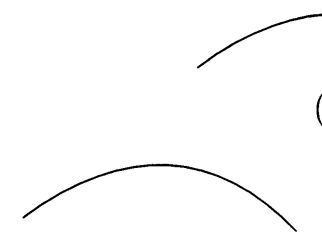

(2)

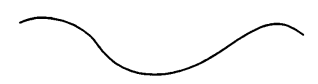

(4)

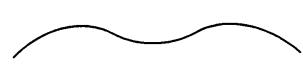

(6)

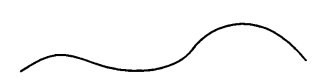

(8)

(1)

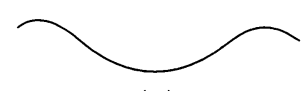

(3)

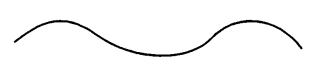

(5)

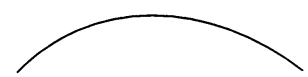

(7)

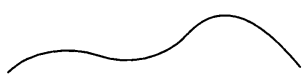

(9)

$$
\alpha=\pi / 4
$$

FIG. 3.2

In a couple of the equilibria (cf. Fig. (3.4) and Fig. (3.5)) the arch has actually passed through itself. In reality this would not happen and the actual equilibria would have be determined by analyzing the contact problem. This type of analysis has been done in the case of the circular ring under normal pressure (cf. [6]).

\begin{tabular}{|l|l|l|l|l|l|l|l|l|l|}
\hline & \multicolumn{3}{|c|}{$\alpha=\pi / 4$} & \multicolumn{3}{c|}{$\alpha=\pi / 2$} & \multicolumn{3}{c|}{$\alpha=3 \pi / 4$} \\
\hline & $\lambda$ & $\mu$ & $\omega$ & $\lambda$ & $\mu$ & $\omega$ & $\lambda$ & $\mu$ & $\omega$ \\
\hline 1 & 0 & 0 & 0 & 0 & 0 & 0 & 0 & 0 & 0 \\
\hline 2 & -18 & 16.55 & 0 & -5 & 3.30 & 0 & -3.5 & .41 & 0 \\
\hline 3 & -18 & 16.69 & 0 & -5 & 5.93 & 0 & -3.5 & 3.95 & 0 \\
\hline 4 & -30 & 6.79 & 0 & -10 & 2.80 & 0 & -8.0 & 2.87 & 0 \\
\hline 5 & -7 & 32.84 & 0 & -2.2 & 9.5 & 0 & -1.5 & 5.50 & 0 \\
\hline 6 & -20 & 43.72 & 0 & -5 & 11.30 & 0 & -3.0 & 5.55 & 0 \\
\hline 7 & -40 & 38.10 & 0 & -9 & 6.65 & 0 & -5.6 & 1.90 & 0 \\
\hline 8 & -15 & 33.87 & -2.6 & -5 & 9.37 & -.8 & -3.0 & 4.90 & .37 \\
\hline 9 & -70 & 33.47 & -2.7 & -8 & 7.17 & .55 & -8.0 & 1.52 & 2.87 \\
\hline
\end{tabular}




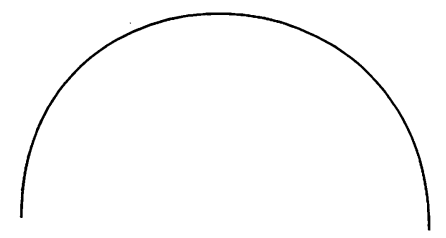

(1)

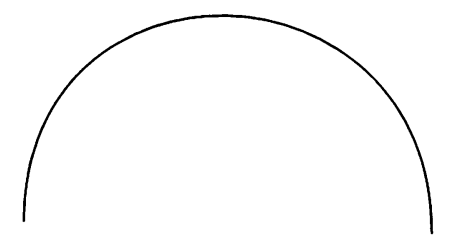

(2)

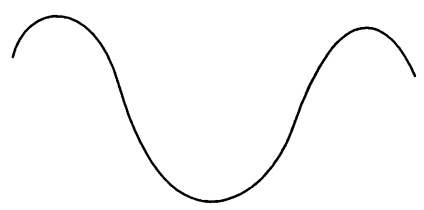

(4)

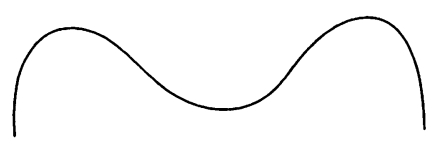

(6)

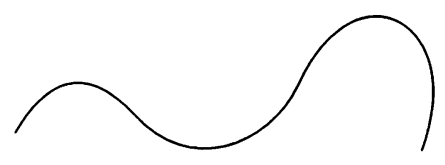

(8)

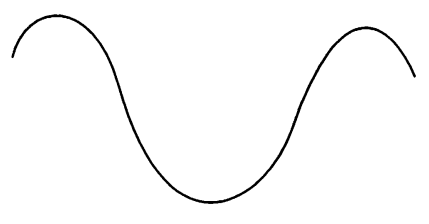

(3)

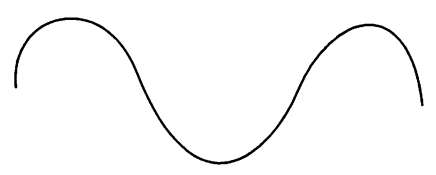

(5)

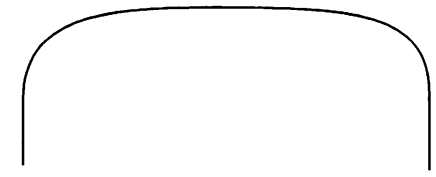

(7)

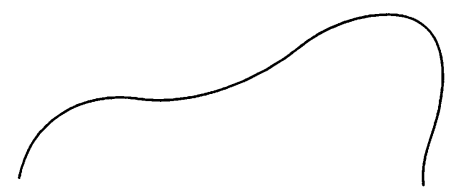

(9)

$$
\alpha=\pi / 2
$$

FIG. 3.3 


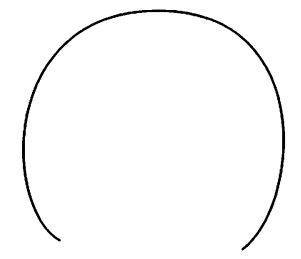

(1)

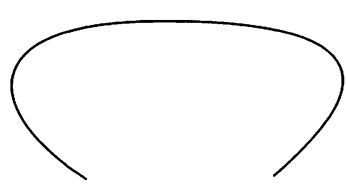

(2)

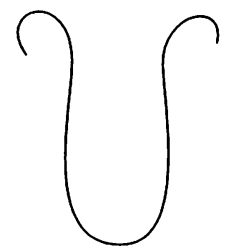

(4)

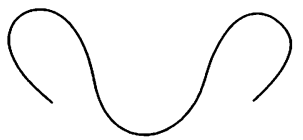

(6)

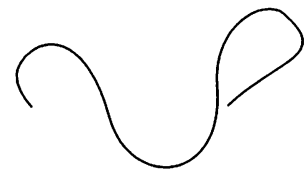

(8)

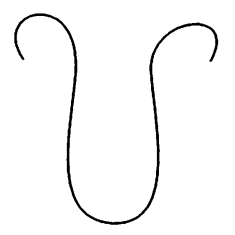

(3)

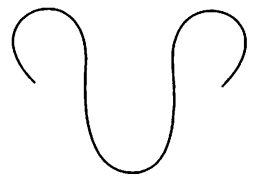

(5)

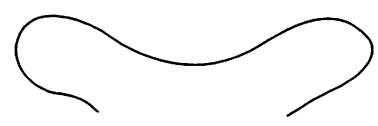

(7)

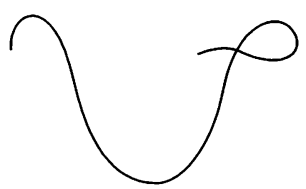

(9)

$\alpha=3 \pi / 4$

FIG. 3.4 

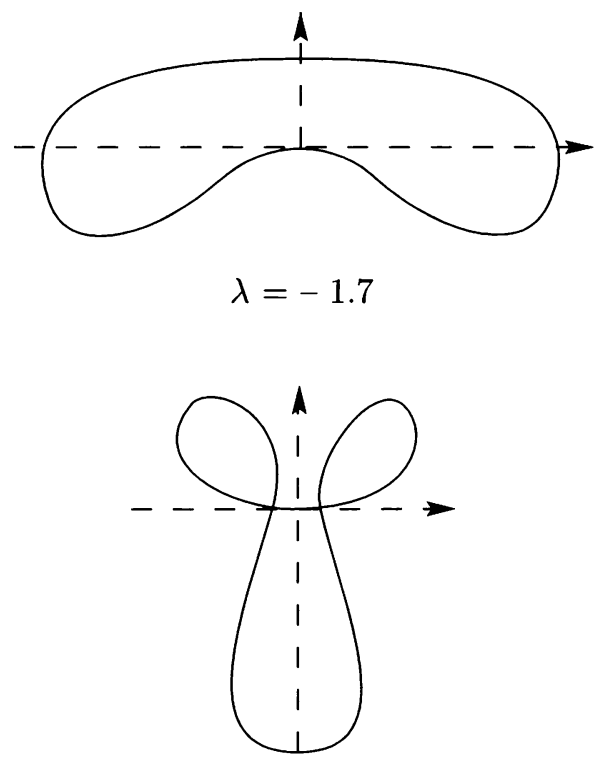

$\lambda=-2$

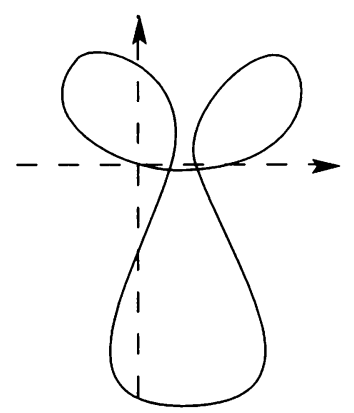

$$
\begin{gathered}
\lambda=-2.5 \\
\alpha=\pi
\end{gathered}
$$

FIG. 3.5

4. Appendix. Derivation of the equations. We consider a portion of a circular ring whose radius, measured to the center-line, is $a$. Before deformation the position of a point or the center-line is given by

$$
\vec{R}=a \hat{e}_{r}
$$

( $\hat{e}_{r}$ and $\hat{e}_{\theta}$ will denote unit vectors in polar coordinates). After deformation this point has a new position

$$
\vec{R}^{*}=(a+w) \hat{e}_{r}+u \hat{e}_{\theta} .
$$

The functions $w$ and $u$ depend on the polar angle $\theta$ where $-\alpha<\theta<\alpha(0<\alpha<\pi)$. 
Before deformation, the position of a point off the center-line of the arch can be written

$$
\vec{X}=(a-r) \hat{e}_{r}=\vec{R}-r \hat{e}_{r}
$$

In order to describe the position of the point after deformation, we will use the assumptions von Kármán introduced in his study of plates and shells (cf. [7]). In particular, we assume (i) a normal to the center-line before deformation remains normal after deformation and (ii) a point at a distance $r$ from the center-line before deformation remains a distance $r$ after deformation. Thus, after deformation, the point has a new position

$$
\vec{X}^{*}=(a+w) \hat{e}_{r}+u \hat{e}_{\theta}+r \hat{n}
$$

where $\hat{n}$ is the principal normal to the deformed center-line.

For small strains the strain energy of the arch is given by

$$
\mathcal{E}=\int_{A} \int_{-\alpha}^{\alpha} \frac{E}{2} \epsilon^{2} a d \theta d A
$$

where $A$ is the area of a cross section of an element of the arch, $E$ is the Young's Modulus, and $\mathcal{E}$ is the circumferential strain in the arch. The strain energy is dependent on the single strain $\epsilon$ since the von Kármán assumptions guarantee that there are no radial or shear strains. It is convenient to introduce a variable $\psi$ by

$$
\hat{n}=-\left(\cos \psi \hat{e}_{r}+\sin \psi \hat{e}_{\theta}\right)
$$

Thus $\psi$ measures the change in angle of the normal to the center-line after deformation. We will assume the arch is clamped at both ends. Therefore,

$$
\psi(\alpha)=\psi(-\alpha)=0
$$

The theory of arches is a geometrically exact theory in which it is assumed that the strain in the center-line is zero. Under these assumptions, it is shown in [1] that the circumferential strain is given by

$$
\epsilon=-\frac{r}{a-r} \psi^{\prime}
$$

where the prime denotes differentiation with respect to $\theta$. It is also shown in [1] that the displacements $w$ and $u$ are related to $\psi$ by

$$
\begin{aligned}
\frac{w^{\prime}-u}{a} & =-\sin \psi, \\
1+\frac{w+u^{\prime}}{a} & =\cos \psi .
\end{aligned}
$$

Combining (4.5) and (4.8) we find that the strain energy is given by

$$
\mathcal{E}=\int_{-\alpha}^{\alpha} \frac{E I}{2}\left(\psi^{\prime}\right)^{2} a d \theta
$$


when the constant $I$ is given by (1.5). It will be assumed that the arch is acted on by a constant force $P$ in the direction of the negative $x$ axis. Thus the force can be written

$$
\vec{F}=P\left(\cos \theta \hat{e}_{r}-\sin \theta \hat{e}_{\theta}\right)=P \hat{i}
$$

( $\hat{i}$ is the unit vector in the $x$ direction). In the figures in Sec. 3 the arch has been rotated so that the force acts downwards. However, for computational purposes the above formulation is more convenient. In any case the work done by the force in (4.11) is

$$
\begin{aligned}
W & =-\int_{-\alpha}^{\alpha} \vec{F} \cdot\left(w \hat{e}_{r}+u \hat{e}_{\theta}\right) a d \theta \\
& =-\int_{-\alpha}^{\alpha} P(w \cos \theta-u \sin \theta) a d \theta
\end{aligned}
$$

In order to rewrite (4.12) in terms of $\psi$ it is necessary to solve Eqs. (4.9) with the boundary conditions

$$
\begin{gathered}
w(-\alpha)=w(\alpha)=0, \\
u(-\alpha)=u(\alpha)=0 .
\end{gathered}
$$

Since the arch is clamped, $\psi$ satisfies the boundary condition (1.2).

The equations (4.9) imply that $w$ satisfies the second-order equation

$$
\frac{w^{\prime \prime}+w}{a}=-1+\cos \psi-\psi^{\prime} \cos \psi
$$

The general solution (4.14) is

$$
\begin{aligned}
\frac{w}{a}=A & \cos \theta+B \sin \theta \\
& +\int_{-\alpha}^{\theta} \sin (\theta-\xi)\left(-1+\cos \psi-\frac{d}{d \xi} \sin \psi\right) d \xi
\end{aligned}
$$

where $A$ and $B$ are constants to be determined by the boundary conditions. After simplification, Eq. (4.15) can be written

$$
\begin{aligned}
\frac{w}{a}= & A \cos \theta+B \sin \theta+\cos (\alpha+\theta)-1 \\
& +\sin \theta \int_{-\alpha}^{\theta} \cos (\psi+\xi) d \xi-\cos \theta \int_{-\alpha}^{\theta} \sin (\psi+\xi) d \xi
\end{aligned}
$$

The boundary conditions (4.13a) lead to the equations

$$
\begin{aligned}
& A \cos \alpha-B \sin \alpha=0 \\
& A \cos \alpha+B \sin \alpha+\cos 2 \alpha-1 \\
& \quad+\sin \alpha \int_{-\alpha}^{\alpha} \cos (\psi+\xi) d \xi-\cos \alpha \int_{-\alpha}^{\alpha} \sin (\psi+\xi) d \xi=0 .
\end{aligned}
$$


Assuming $\alpha \neq \pi / 2$ and $\alpha \neq \pi$ we find

$$
\begin{aligned}
A= & \frac{1}{2 \cos (\alpha)}\left(2 \sin ^{2} \alpha-\sin \alpha \int_{-\alpha}^{\alpha} \cos (\psi+\xi) d \xi\right. \\
& \left.+\cos \alpha \int_{-\alpha}^{\alpha} \sin (\psi+\xi) d \xi\right) \\
B= & \frac{1}{2 \sin (\alpha)}\left(2 \sin ^{2} \alpha-\sin \alpha \int_{-\alpha}^{\alpha} \cos (\psi+\xi) d \xi\right. \\
& \left.+\cos \alpha \int_{-\alpha}^{\alpha} \sin (\psi+\xi) d \xi\right) .
\end{aligned}
$$

It is a consequence of $(4.9 \mathrm{a})$ that

$$
\begin{aligned}
\frac{u}{a}= & -A \sin \theta+B \cos \theta-\sin (\alpha+\theta) \\
& +\cos \theta \int_{-\alpha}^{\theta} \cos (\psi+\xi) d \xi+\sin \theta \int_{-\alpha}^{\theta} \sin (\psi+\xi) d \xi
\end{aligned}
$$

Using the same argument as above, the boundary conditions (4.13b) imply that

$$
\begin{array}{r}
A=\frac{1}{2 \sin (\alpha)}\left(-\sin (2 \alpha)+\cos \alpha \int_{-\alpha}^{\alpha} \cos (\psi+\xi) d \xi\right. \\
\left.+\sin \alpha \int_{-\alpha}^{\alpha} \sin (\psi+\xi) d \xi\right), \\
B=\frac{1}{2 \cos (\alpha)}\left(\sin 2 \alpha-\cos \alpha \int_{-\alpha}^{\alpha} \cos (\psi+\xi) d \xi\right. \\
\left.-\sin \alpha \int_{-\alpha}^{\alpha} \sin (\psi+\xi) d \xi\right)
\end{array}
$$

$(\alpha \neq \pi / 2, \alpha \neq \pi)$. The values given in (4.18) and (4.20) for the constants $A$ and $B$ must agree. There is no difficulty in showing that a necessary and sufficient condition for this agreement is that the conditions (1.3) be satisfied. The conditions (1.3) are constraints on the variational problem that guarantee that the displacements satisfy the appropriate boundary conditions. In the above remarks we have assumed that $\alpha \neq \pi / 2$ and $\alpha \neq \pi$. However, conditions (1.3) are also correct in these special cases.

An elementary calculation (cf. (4.16) and (4.19)) shows that

$$
\frac{w \cos \theta-u \sin \theta}{a}=A+\cos \alpha-\int_{\alpha}^{\theta} \sin (\psi+\xi) d \xi .
$$

The only term in (4.21) that will make a contribution to the Euler equation for the variational problem is the integral term. Therefore, it suffices to choose the work (cf. 
(4.12)) to be

$$
\begin{aligned}
W & =\int_{-\alpha}^{\alpha} P\left(\int_{-\alpha}^{\theta} \sin (\psi+\xi) d \xi\right) a^{2} d \theta \\
& =P \alpha a^{2} \int_{-\alpha}^{\alpha} \sin (\psi+\theta) d \theta-a^{2} \int_{-\alpha}^{\alpha} P \theta \sin (\psi+\theta) d \theta
\end{aligned}
$$

Standard arguments (cf. [8]) imply that the equilibria of the arch are the stationary values of $\mathcal{E}-W$ subject to the constraints (1.3) and the boundary conditions (1.2). Equivalently, the equilibrium equation is the Euler equation for the integral

$$
\int_{-\alpha}^{\alpha}\left\{\frac{E I}{2}\left(\psi^{\prime}\right)^{2}-P a \theta \sin (\theta+\xi)+\mu_{1} \cos (\psi+\theta)+\mu_{2} \sin (\psi+\theta)\right\} \varphi d \theta .
$$

Equation (1.1) is a consequence of (4.23).

\section{REFERENCES}

[1] R. W. Dickey and J. J. Roseman, Equilibria of the circular elastica under a uniform central force field, Quart. Appl. Math. 51, 201-216 (1993)

[2] I. Tadjbaksh and F. Odeh, Equilibrium states of elastic rings, J. Math. Anal. Appl. 18, 59-74 (1967)

[3] S. S. Antman, A note on a paper of Tadjbaksh and Odeh, J. Math. Anal. Appl. 21, 132-135 (1968)

[4] J. J. Stoker, Nonlinear Elasticity, Gordon and Breach, New York, 1968

[5] E. Isaacson and H. B. Keller, Analysis of Numerical Methods, John Wiley and Sons, Inc., New York, 1966

[6] J. E. Flaherty, J. B. Keller, and S. I. Rubinow, Post buckling behavior of elastic tubes and rings with opposite sides in contact, SIAM J. Appl. Math. 23, 446-455 (1972)

[7] T. von Kármán, Festigkeitsprobleme im Maschinenbau, Enzyklopädie der Mathematischen Wissenschaften, IV-4, Leipzig, 1910

[8] I. M. Gelfand and S. V. Fomin, Calculus of Variations, Prentice-Hall, Inc., Englewood Cliffs, NJ, 1963

[9] S. S. Antman and J. E. Dunn, Qualitative behavior of buckled nonlinearly elastic arches, J. Elasticity 10, 225-239 (1980)

[10] S. O. Asplund, Structural Mechanics: Classical and Matrix Methods, Prentice-Hall, Inc., Englewood Cliffs, NJ, 1966 